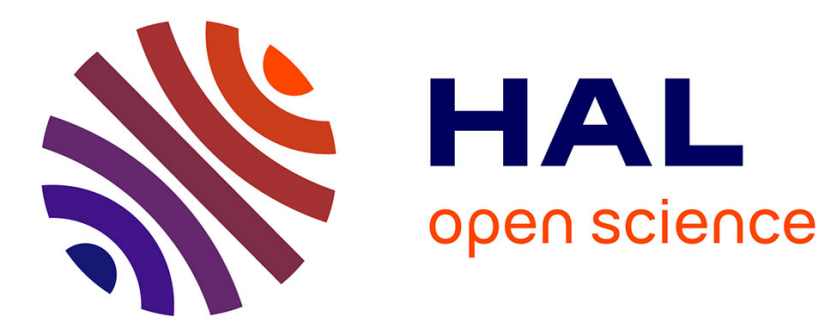

\title{
An exact Riemann solver for multicomponent turbulent flow
}

\author{
Emmanuelle Declercq, Alain Forestier, Jean-Marc Hérard, Xavier Louis, \\ Gérard Poissant
}

\section{- To cite this version:}

Emmanuelle Declercq, Alain Forestier, Jean-Marc Hérard, Xavier Louis, Gérard Poissant. An exact Riemann solver for multicomponent turbulent flow. International Journal of Computational Fluid Dynamics, 2001, 14, pp.117-131. 10.1080/10618560008940719 . hal-01580049

\section{HAL Id: hal-01580049 https://hal.science/hal-01580049}

Submitted on 23 Dec 2019

HAL is a multi-disciplinary open access archive for the deposit and dissemination of scientific research documents, whether they are published or not. The documents may come from teaching and research institutions in France or abroad, or from public or private research centers.
L'archive ouverte pluridisciplinaire $\mathbf{H A L}$, est destinée au dépôt et à la diffusion de documents scientifiques de niveau recherche, publiés ou non, émanant des établissements d'enseignement et de recherche français ou étrangers, des laboratoires publics ou privés. 


\title{
Part I
}

\section{An exact Riemann solver for a multicomponent turbulent flow.}

\author{
Emmanuelle Declercq *† Alain Forestier ${ }^{*} \quad$ Jean-Marc Hérard $\mathbb{N} \mid \quad$ Xavier Louis** \\ Gérard Poissant ${ }^{\dagger \dagger}$
}

key words- Multicomponent turbulence model - Entropy characterization - Riemann solver.

abstract- This contribution's topic is the resolution of the hyperbolic system which describes a multicomponent turbulent flow. The model is written for an isentropic gas. We compute the exact solution of the Riemann Problem (RP) associated to the hyperbolic system. It is composed of constant states separated by rarefaction waves, or shock waves and a contact discontinuity. The selection of the admissible part of the shock curve is obtained by an entropic criterion. Compressive shock means entropic shock for only one of the two mathematical entropies found. This entropy is the total energy of the system. With these existence and uniqueness properties, we compute the exact solution of (RP) by a Smoller's kind of parameterization.

\section{Introduction}

The recent need for computation of complex systems of non linear PDE's such as those arising when investigating turbulent phenomena has motivated the development of adequate solvers. Actually hyperbolic systems arising in the framework of single phase turbulent compressible

\footnotetext{
${ }^{*}$ C.E.M.I.F., 40 rue du Pelvoux, Courcouronnes, 91020 Evry Cedex (France) declercq@worldonline.fr

${ }^{\dagger}$ C.E.A. Saclay, DRN/DMT/SEMT, 91191 Gif-Sur-Yvette Cedex (France) xaviern@semt2.smts.cea.fr

${ }^{\ddagger}$ C.E.A. Saclay, DRN/DMT/SEMT, 91191 Gif-Sur-Yvette Cedex (France) alain.forestier@cea.fr

${ }^{\S}$ C.E.M.I.F., 40 rue du Pelvoux, Courcouronnes, 91020 Evry Cedex (France)

"E.D.F. LNH/DER, 6 quai Watier, 78400 Chatou (France) Jean-Marc.Herard@der.edf.fr

"C.M.I UMR CNRS 6632, Université de Provence, 39 rue Joliot Curie, 13453 Marseille

** C.E.A. Saclay, DRN/DMT/SEMT, 91191 Gif-Sur-Yvette Cedex (France) for@semt2.smts.cea.fr

${ }^{\dagger \dagger}$ C.E.M.I.F., I.U.T. G.M.P., 3 cours Mgr Roméro, 91020 Evry (France) G.Poissant@iut.univ-evry.fr
} 
models contain different scales of pressure fields. The standard mean pressure accounts for microscopic effects, whereas the mean turbulent kinetic energy (focusing on K-epsilon type models) stands for some counterpart of the mean pressure at a macroscopic level. This was recently demonstrated by several workers (see for instance Coquel and Berthon [1], or [2]) who hence proposed various upwinding schemes for practical purposes. This is true for one or two-equation models, but it is even more convincing when turning to so-called second-moment closures. In this case, the very small amount of viscous effects urges investigating basic solutions of homogeneous convective systems. Though the decoupled approaches are still often used in industrial codes, recent examples of computation of impinging jets on wall boundaries have shown that the coupled approach should be preferred for stability reasons. We will focus in this work on the tight coupling between the mean pressure field and turbulent kinetic energy, when computing multi-component compressible first-order turbulent closures. One of the main objectives here is to derive exact or approximate Riemann solvers for our specific problem, and beyond to compare both efficiency, accuracy and stability of respective schemes. The paper is thus organized as follows. In the first part, the turbulent model used to describe the flow is briefly presented. Since both viscous and source terms may be easily computed applying standard Finite Volume schemes on structured meshes at least, emphasis is given on the analysis of the convective homogeneous problem, which is hyperbolic but is not under conservative form. Studying Riemann invariants, entropy inequality and assuming some approximate jump conditions hold, enables to derive an existence and uniqueness result for the solution of the one-dimensional Riemann problem associated with the convective problem, provided that the initial data agrees with some condition. This result is made possible by using the admissible part of the shock curves owing to the entropy inequality. It also requires that the strength of shocks is sufficiently weak.

The second part of the paper is devoted to the construction of a Godunov type solver which accounts for non-conservative terms, and to comparison with some rough Godunov scheme, and also with the adaptation to the frame of non conservative systems of the rough but robust Rusanov scheme. 


\section{A turbulence model to describe multicomponent flows}

\subsection{Governing equations}

We begin with Euler equations for an average compressive multicomponent flow (see [9]). The gas $k=v, l$ are assumed to be isentropic like in the P-system. We define by $\bar{\rho}$ the mean density of the mixture, $\alpha$ the volume fraction of the $v$ flow in the mixture, $P$ the pressure and $U$ the velocity of the mixture. We use Favre's average [11] to deal with compressive flows :

$$
\begin{gathered}
\overline{\rho_{k} U_{k}}=\bar{\rho}_{k} \tilde{U}_{k}=\alpha_{k} \overline{\bar{\rho}}_{k} \tilde{U}_{k} \quad k=v, l \\
\alpha=\alpha_{v}, \quad \bar{\rho}=\alpha \overline{\bar{\rho}}_{v}+(1-\alpha) \overline{\bar{\rho}}_{l}, \quad \bar{\rho} \tilde{U}=\alpha \overline{\bar{\rho}}_{v} \tilde{U}_{v}+(1-\alpha) \overline{\bar{\rho}}_{l} \tilde{U}_{l}
\end{gathered}
$$

We introduce the mass fraction $Y$, and the relative velocity $V_{k}$ :

$$
\begin{gathered}
Y=Y_{v}=\frac{\alpha \overline{\overline{\rho_{v}}}}{\bar{\rho}}, \quad V_{k}=\tilde{U}_{k}-\tilde{U}, \quad \sum_{k} Y_{k} V_{k}=Y V_{v}+(1-Y) V_{l}=0 \\
\sum_{k} Y_{k} V_{k}^{2}=Y \frac{V_{v}^{2}}{1-Y} \Rightarrow \alpha \overline{\bar{\rho}}_{v} \tilde{U}_{v}^{2}+(1-\alpha) \overline{\bar{\rho}}_{l} \tilde{U}_{l}^{2}=\bar{\rho} \tilde{U}^{2}+\bar{\rho} Y \frac{V_{v}^{2}}{1-Y} \\
(\overline{\mathcal{S}})\left\{\begin{array}{l}
\partial_{t}(\bar{\rho})+\nabla(\bar{\rho} \tilde{U})=0 \\
\partial_{t}(\bar{\rho} Y)+\nabla(\bar{\rho} Y \tilde{U})=-\nabla\left(\bar{\rho} Y V_{v}\right) \\
\partial_{t}(\bar{\rho} \tilde{U})+\nabla\left(\bar{\rho} \tilde{U}^{2}+\frac{2}{3} K^{s}+\overline{\bar{P}} \overline{\bar{I}}\right)=-\nabla\left(\bar{\rho} Y \frac{V_{v}^{2}}{1-Y}\right)
\end{array}\right.
\end{gathered}
$$

The kinetic turbulent energy $K^{v}$ is the trace of the $\frac{1}{2} \overline{\overline{R^{v}}}$ tensor. In the two dimensional frame we write :

$$
2 K^{v}=\operatorname{Tr}(\overline{\bar{R}})=\sum_{i} \overline{\bar{R}}_{i i}=\overline{\rho_{v}\left(u_{v}^{2}+v_{v}^{2}\right)}-\alpha \overline{\overline{\rho_{v}}}\left(\tilde{u}_{v}^{2}+\tilde{v}_{v}^{2}\right)
$$

Remark that we have noted $K^{s}=K^{v}+K^{l}=\frac{1}{2}\left(\overline{\rho_{v} U_{v}^{\prime 2}}+\overline{\rho_{l} U_{l}^{\prime 2}}\right)$

But that is not the turbulence of the melting flow $K=\frac{1}{2} \overline{\rho U^{2}}$

\subsection{K model for isentropic multicomponents flows}

To close the model we derive a supplementary equation for the kinetic turbulent energy in the $v$ flow. To compute this equation we subtract the equation of $\alpha \overline{\overline{\rho_{v}}} \tilde{U}_{v}^{2}$ from the equation of $\overline{\rho_{v} U_{v}^{2}}$. 
We introduce the deviator $\overline{\bar{D}}$ such that :

$$
\overline{\bar{R}}=\frac{\operatorname{Tr}(\overline{\bar{R}})}{3} \overline{\bar{I}}+\overline{\bar{D}}
$$

\section{Proposition 1}

The evolution equation of a discontinuous by phases turbulent flow is :

$$
\partial_{t}\left(K^{v}\right)+\nabla \cdot\left(K^{v} \tilde{U}_{v}\right)+\frac{2}{3} K^{v} \nabla \tilde{U}_{v}-2\left(\nabla^{t} \overline{\bar{D}}\right) \tilde{U}_{v}+\nabla \overline{\rho_{v} U_{v}^{\prime 3}}+\overline{U_{v}^{\prime} \nabla P}+\overline{\rho_{v} U_{v}^{\prime 2}\left(U_{v}-v_{i}\right) a_{i} n_{v}}=0
$$

$\underline{\text { Proof : }}$

$$
\begin{aligned}
& \partial_{t}\left(\rho_{v} U_{v}^{2}\right)+\nabla\left(\rho_{v} U_{v}^{3}\right)+2 U_{v} \nabla(P \overline{\bar{I}})=0 \\
& \partial_{t}\left(\overline{\rho_{v} U_{v}^{2}}\right)+\nabla\left(\overline{\rho_{v} U_{v}^{3}}\right)+\overline{2 U_{v} \nabla(P \overline{\bar{I}})}+\overline{\rho_{v} U_{v}^{2}\left(U_{v}-v_{i}\right) a_{i} n_{v}}=0 \\
& \partial_{t}\left(\alpha \overline{\overline{\rho_{v}}} \tilde{U}_{v}{ }^{2}\right)+\nabla\left(\alpha \overline{\overline{\rho_{v}}} \tilde{U}_{v}{ }^{3}\right)+2\left(\nabla^{t} \overline{\overline{R_{v}}}\right) \tilde{U}_{v}+2 \alpha \tilde{U}_{v} \nabla(P \overline{\bar{I}})-2 \tilde{U}_{v} M_{v}^{d}=0 \\
& 2 K^{v}=\operatorname{tr}\left(\overline{\overline{R^{v}}}\right)=\overline{\rho_{v} U_{v}^{\prime 2}}=\overline{\rho_{v} U_{v}^{2}}-\alpha \overline{\overline{\rho_{v}}} \tilde{U}_{v}^{2} \\
& \left.\partial_{t}\left(2 K^{v}\right)+\nabla\left(\overline{\rho_{v} U_{v}^{3}}-\alpha \overline{\overline{\rho_{v}}} \tilde{U}_{v}^{3}\right)-2\left(\nabla^{t} \overline{\bar{R}}^{v}\right) \tilde{U}_{v}+2 \overline{U_{v} \nabla(P \overline{\bar{I}}}\right)-2 \alpha \tilde{U}_{v} \nabla(P \overline{\bar{I}}) \\
& +\overline{\rho_{v} U_{v}^{2}\left(U_{v}-v_{i}\right) a_{i} n_{v}}-2 \tilde{U}_{v} \overline{\rho_{v} U_{v}\left(U_{v}-v_{i}\right) a_{i} n_{v}}-\overline{P_{v} a_{i} n_{v}}-\overline{\overline{P_{v}}} \nabla \alpha=0 \\
& \overline{\rho_{v} U_{v}^{3}}-\alpha \overline{\overline{\rho_{v}}} \tilde{U}_{v}{ }^{3}=\overline{\rho_{v} U_{v}^{\prime 3}}+3 \tilde{U}_{v} \overline{\rho_{v} U_{v}^{\prime 2}}=\overline{\rho_{v} U_{v}^{\prime 3}}+3 \operatorname{Tr}\left(\overline{\bar{R}}^{v}\right) \tilde{U}_{v} \\
& \overline{U_{v} \nabla P}=\overline{U_{v}^{\prime} \nabla P}+\tilde{U} \overline{\nabla P}=\overline{U_{v}^{\prime} \nabla P}+\alpha \tilde{U}_{v} \nabla P \tilde{U}_{v} \overline{\bar{P}} \nabla \alpha+\tilde{U}_{v} \overline{P a_{i} n_{v}} \\
& \overline{\rho_{v} U_{v}^{2}\left(U_{v}-v_{i}\right) a_{i} n_{v}}-2 \tilde{U}_{v} \overline{\rho_{v} U_{v}\left(U_{v}-v_{i}\right) a_{i} n_{v}}=\overline{\rho_{v} U_{v}^{\prime 2}\left(U_{v}-v_{i}\right) a_{i} n_{v}}-\tilde{U}_{v}^{2} \underbrace{\overline{\rho_{v}\left(U_{v}-v_{i}\right) a_{i} n_{v}}}_{\Gamma_{v} \approx 0} \\
& \left.\partial_{t}\left(2 K^{v}\right)+\nabla\left(3 \operatorname{Tr}\left(\overline{\overline{R^{v}}}\right) \tilde{U}_{v}\right)-2\left(\nabla^{t} \overline{\overline{R_{v}}}\right)\right) \tilde{U}_{v}+\nabla \overline{\rho_{v} U_{v}^{\prime 3}}+\overline{U_{v}^{\prime} \nabla P}+\overline{\rho_{v} U_{v}^{\prime 2}\left(U_{v}-v_{i}\right) a_{i} n_{v}}=0 \\
& \left.\nabla\left(3 \operatorname{Tr}\left(\overline{\overline{R^{v}}}\right) \tilde{U}_{v}\right)-2\left(\nabla^{t} \overline{\overline{R^{v}}}\right)\right) \tilde{U}_{v}=3 \nabla\left(\operatorname{Tr}\left(\overline{\overline{R^{v}}}\right) \tilde{U}_{v}\right)-2 \nabla\left(\frac{\operatorname{Tr}\left(\overline{\overline{R^{v}}}\right)}{3}\right) \cdot \tilde{U}_{v}-2\left(\nabla^{t} \overline{\bar{D}}\right) \tilde{U}_{v} \\
& =\operatorname{Tr}\left(\overline{\overline{R^{v}}}\right) \nabla \cdot \tilde{U}_{v}+\nabla\left(\operatorname{Tr}\left(\overline{\overline{R^{v}}}\right) \tilde{U}_{v}+\frac{2}{3} \operatorname{Tr}(\overline{\bar{R}}) \nabla \cdot \tilde{U}_{v}-2\left(\nabla^{t} \overline{\bar{D}}\right) \tilde{U}_{v}\right. \\
& =\nabla\left(2 K^{v} \tilde{U}_{v}\right)+\frac{4 K^{v}}{3} \nabla \cdot \tilde{U}_{v}-\left(\nabla^{t} \overline{\bar{D}}\right) \tilde{U}_{v} \\
& \partial_{t}\left(K^{v}\right)+\nabla\left(K^{v} \tilde{U}_{v}\right)+\frac{2 K^{v}}{3} \nabla \cdot \tilde{U}_{v}-2\left(\nabla^{t} \overline{\bar{D}}\right) \tilde{U}_{v}+\nabla \overline{\rho_{v} U_{v}^{\prime 3}}+\overline{U_{v}^{\prime} \nabla P}+\overline{\rho_{v} U_{v}^{\prime 2}\left(U_{v}-v_{i}\right) a_{i} n_{v}}=0
\end{aligned}
$$


Then we make some simplifications to close the system. At first we neglect area source terms, and odd correlations.

$$
\begin{gathered}
\overline{\rho_{v} U_{v}^{\prime 2}\left(U_{v}-v_{i}\right) a_{i} n_{v}} \approx 0 \\
\nabla \overline{\rho_{v} U_{v}^{\prime 3}} \approx 0
\end{gathered}
$$

After, we assume an isotropic turbulence, so the Reynolds tensor is diagonal and isotropic. It is described through $K^{v}$ :

$$
R_{i j}^{v}=\frac{2}{3} K^{v} \delta_{i j}
$$

In a two dimensional framework we obtain :

$$
\partial_{t}\left(K^{k}\right)+\nabla\left(K^{k} \tilde{U}_{v}\right)+\frac{2}{3} K^{k} \nabla \tilde{U}_{v}+\overline{U^{\prime} \nabla P}=0
$$

To close the $(\mathcal{S})$ system we add the $K^{s}$ evolution equation. This one is obtained by summation of $K^{k}$ over phases. We suppose that the flows have the same velocity. ¿From now on, we neglect the average symbol, and set $K$ for $K^{s}$. We give the system here obtained adding the viscous terms ( $\mu_{t}$ and $\eta$ are positive quantities depending on the choice of the turbulence model, $\left.\mu_{e f f}=\mu_{\text {lam }}+\mu_{t}\right)$. We recall that the melting gas is isentropic, with a pressure law $P(\rho Y)$ known. $\varepsilon$ is the turbulent dissipation which is modeled (see for example the one equation turbulence model of $[16]$ or [3]).

$$
(\mathcal{S})\left\{\begin{array}{l}
\partial_{t} \rho+\nabla \cdot(\rho U)=0 \\
\partial_{t}(\rho Y)+\nabla \cdot(\rho Y U)=0 \\
\partial_{t}(\rho U)+\nabla \cdot\left(\rho U^{2}+\left(\frac{2}{3} K+P\right) \overline{\bar{I}}\right)=\nabla \cdot\left(\mu_{e f f}\left(\nabla U+\nabla U^{t}-\frac{2}{3}(\nabla \cdot U) \overline{\bar{I}}\right)\right. \\
\partial_{t} K+\nabla \cdot(K U)+\frac{2}{3} K \nabla \cdot U=\mu_{t}\left(\left(\nabla U+\nabla U^{t}-\frac{2}{3}(\nabla \cdot U) \overline{\bar{I}}\right): \nabla U\right)+\nabla\left(\eta \nabla\left(\frac{K}{\rho}\right)\right)-\rho \varepsilon
\end{array}\right.
$$

Setting $W=(C, K)$, we are interested in the first order convective system $\left(\mathcal{S}_{c}\right)$ which is conservative in $C(\rho, \rho Y, \rho U)$ variable, but not in $K$ variable:

$$
\left(\mathcal{S}_{c}\right)\left\{\begin{array}{l}
\partial_{t} C+\nabla F(C, K)=0 \\
\partial_{t} K+\nabla(K U)+\frac{2}{3} K \nabla U=0
\end{array}\right.
$$




\section{Exact Riemann solver}

\subsection{From a 3D problem to the 1D Riemann Problem}

It is well known that Finite Volume upwinding schemes are efficient methods to solve no linear hyperbolic systems. The most natural finite volume method is the Godunov's method [14] which requires getting the exact solution of the Riemann Problem at the interface between two neighboring cells. However, unless the initial data for the turbulent kinetic energy $\mathrm{K}$ is null, the Riemann solution of the multidimensional $(\mathcal{S})$ system is unknown. Hence one needs to exhibit the one dimensional solution of the Riemann problem associated with the whole convective terms. The 1D associated problem is a differential system in the normal direction of the boundaries of a two dimensional control volume.

$$
\left(\mathcal{S}_{c}\right) \quad \partial_{t} W+A \partial_{x} W+B \partial_{y} W=0
$$

\section{Proposition 2}

The $W_{n}$ solution of $\left(\mathcal{S}_{n}\right)$ is the normal projection of the $W$ solution of the $(\mathcal{S})$ system.

$$
\begin{gathered}
\left(\mathcal{S}_{n}\right) \quad \partial_{t} W_{n}+N \partial_{n} W_{n}=0 \\
\text { Noting } N=\left(P A P^{-1}\right) \cdot n_{x}+\left(P B P^{-1}\right) \cdot n_{y} \\
W_{n}\left(\begin{array}{c}
Y \\
\rho \\
u_{n} \\
u_{\tau} \\
K
\end{array}\right) \quad \vec{n}=\left(\begin{array}{c}
n_{x} \\
n_{y}
\end{array}\right) \quad \vec{\tau}=\left(\begin{array}{c}
-n_{y} \\
n_{x}
\end{array}\right) \overrightarrow{U_{n}}=\left(\begin{array}{l}
u_{n}=u \cdot n_{x}+v \cdot n_{y} \\
u_{\tau}=-u \cdot n_{y}+v \cdot n_{x}
\end{array}\right)
\end{gathered}
$$

With $\tilde{c}$ the celerity in the multicomponent flow : $\tilde{c}^{2}=P^{\prime}(\rho Y)$

in the multicomponent flow :

$$
A=\left(\begin{array}{ccccc}
u & 0 & 0 & 0 & 0 \\
0 & u & \rho & 0 & 0 \\
\tilde{c}^{2} & \frac{Y \tilde{c}^{2}}{\rho} & u & 0 & \frac{2}{3 \rho} \\
0 & 0 & 0 & u & 0 \\
0 & 0 & \frac{5}{3} & 0 & u
\end{array}\right) \quad B=\left(\begin{array}{ccccc}
v & 0 & 0 & 0 & 0 \\
0 & v & 0 & \rho & 0 \\
0 & 0 & v & 0 & 0 \\
\tilde{c}^{2} & \frac{Y \tilde{c}^{2}}{\rho} & 0 & v & \frac{2}{3 \rho} \\
0 & 0 & 0 & \frac{5}{3} & v
\end{array}\right) \quad P=\left(\begin{array}{ccccc}
1 & 0 & 0 & 0 & 0 \\
0 & 1 & 0 & 0 & 0 \\
0 & 0 & n_{x} & n_{y} & 0 \\
0 & 0 & n_{y} & -n_{x} & 0 \\
0 & 0 & 0 & 0 & 1
\end{array}\right)
$$


Applying the $P$ projector to the $(\mathcal{S})$ system :

$$
\partial_{t} W_{n}+P A \partial_{x} W+P B \partial_{y} W=0
$$

Thus, using the fact that: $W=P^{-1} W_{n}$ enables to derive :

$$
\begin{gathered}
\partial_{t} W_{n}+P A P^{-1} \partial_{x}(P W)+P B P^{-1} \partial_{y}(P W)=0 \\
\partial_{t} W_{n}+P A P^{-1} \partial_{x}\left(W^{n}\right)+P B P^{-1} \partial_{y}\left(W^{n}\right)=0 \\
\quad \begin{array}{l}
N n_{x}=P A P^{-1} \\
N n_{y}=P B P^{-1}
\end{array} \\
N=\left(P A P^{-1}\right) \cdot n_{x}+\left(P B P^{-1}\right) \cdot n_{y}=\left(\begin{array}{ccccc}
u_{n} & 0 & 0 & 0 & 0 \\
0 & u_{n} & \rho & 0 & 0 \\
\tilde{c}^{2} & \frac{Y \tilde{c}^{2}}{\rho} & u_{n} & 0 & \frac{2}{3 \rho} \\
0 & 0 & 0 & u_{n} & 0 \\
0 & 0 & \frac{5 K}{3} & 0 & u_{n}
\end{array}\right)
\end{gathered}
$$

We eventually obtain a similar one dimensional system :

$$
\left(\mathcal{S}_{n}\right)=\left\{\begin{array}{l}
\partial_{t}(\rho Y)+\partial_{n}\left(\rho Y u_{n}\right)=0 \\
\partial_{t} \rho+\partial_{n}\left(\rho u_{n}\right)=0 \\
\partial_{t}\left(\rho u_{n}\right)+\partial_{n}\left(\rho u_{n}^{2}+\frac{2}{3} K+P\right)=0 \\
\partial_{t}\left(\rho u_{\tau}\right)+\partial_{n}\left(\rho u_{\tau} u_{n}\right)=0 \\
\partial_{t} K+\partial_{n}\left(K u_{n}\right)+\frac{2}{3} K \partial_{n} u_{n}=0
\end{array}\right.
$$

We set by $(P R)$ the $\left(\mathcal{S}_{n}\right)$ associated Riemann Problem with the initial constant states $W_{l}$ and $W_{r}$ on the left and right sides of a the interface.

$$
(P R)\left\{\begin{array}{cc}
\partial_{t} W_{n}+N \partial_{n} W_{n}=0 & \left(\mathcal{S}_{n}\right) \\
W_{n}(X, t=0)=W_{l} \text { if } X . n<0 & \text { I. C. } \\
W_{n}(X, t=0)=W_{r} \text { if } X . n>0 &
\end{array}\right.
$$




\subsection{Exact solution of the 1D Riemann Problem with approximate jump con- ditions}

\subsubsection{Mathematical analysis of the hyperbolic system}

The approach given below is quite similar to analysis of hyperbolic systems occurring in the modeling of spray dynamics ([20], [19]), or of a multicomponent flow in velocity disequilibrium ([17]), of some gas-solid flow models ([5]), or in [18] for a monocomponent turbulent flow. In order to compute the solution of the $(P R)$ problem, we need to investigate the 1 D system $\left(\mathcal{S}_{n}\right)$.

$$
\left(\mathcal{S}_{n}\right) \quad \partial_{t} W_{n}+N \partial_{n} W_{n}=0
$$

The $\left(\mathcal{S}_{n}\right)$ system is hyperbolic, nonstrictly, because the $N$ matrix is diagonalizable in $\mathbb{R}$.

$$
\begin{gathered}
N=\left(\begin{array}{ccccc}
u_{n} & 0 & 0 & 0 & 0 \\
0 & u_{n} & \rho & 0 & 0 \\
\tilde{c}^{2} & \frac{Y \tilde{c}^{2}}{\rho} & u_{n} & 0 & \frac{2}{3 \rho} \\
0 & 0 & 0 & u_{n} & 0 \\
0 & 0 & \frac{5 K}{3} & 0 & u_{n}
\end{array}\right) \\
\operatorname{det}\left(N-\lambda I_{5}\right)=\left(u_{n}-\lambda\right)^{3}\left(u_{n}-\lambda-\tilde{c}^{\prime}\right)\left(u_{n}-\lambda+\tilde{c}^{\prime}\right)
\end{gathered}
$$

We introduce the turbulent celerity in a turbulent multicomponent flow setting:

$$
\left(\tilde{c}^{\prime}\right)^{2}=Y \tilde{c}^{2}+\frac{10}{9} \frac{K}{\rho}
$$

The following eigenvalues quickly arise :

$$
\lambda_{1}=u_{n}-\tilde{c}^{\prime}, \quad \lambda_{2}=\lambda_{3}=\lambda_{4}=u_{n}, \quad \lambda_{5}=u_{n}+\tilde{c}^{\prime}
$$

The associated right eigenvectors span $\mathbb{R}^{5}$ :

$$
\begin{gathered}
r_{1}^{t}=\left(0, \rho,-\tilde{c}^{\prime}, 0, \frac{5}{3} K\right), \quad r_{2}^{t}=(-Y, \rho, 0,0,0), \quad r_{3}^{t}=\left(-1,0,0,0, \frac{3 \rho \tilde{c}^{2}}{2}\right) \\
r_{4}^{t}=(0,0,0,1,0), \quad r_{5}^{t}=\left(0, \rho, \tilde{c}^{\prime}, 0, \frac{5}{3} K\right)
\end{gathered}
$$

The first and fifth characteristic fields are Genuinely Non Linear under sufficient condition that pressure, for fixed $Y$, is a convex function of $\frac{1}{\rho}$ (specific volume) :

$$
2 P^{\prime}(\rho Y)+\rho Y P^{\prime \prime}(\rho Y)>0 \Rightarrow \nabla \lambda_{1} \cdot r_{1}^{t}<0 \text { and } \nabla \lambda_{5} . r_{5}^{t}>0
$$


The field associated with treble eigenvalue is Linearly Degenerated :

$$
\nabla \lambda_{2} \cdot r_{2}^{t}=\nabla \lambda_{3} \cdot r_{3}^{t}=\nabla \lambda_{4} \cdot r_{4}^{t}=0
$$

We are now able to provide the construction of the different smooth waves:

- The simple waves are self-similar solutions, $W_{n}(x, t)=s\left(\frac{x}{t}\right)$ with $x=X . n,(36)$ gives :

$$
\left(N-\frac{x}{t} I\right) s^{\prime}\left(\frac{x}{t}\right)=0
$$

The $j$ simple wave in the domain $\lambda_{j}\left(W_{l}\right) \leq \frac{x}{t} \leq \lambda_{j}\left(W_{r}\right)$ is the integral curve solution of the system :

$$
\left\{\begin{array}{l}
s^{\prime}\left(\frac{x}{t}\right)=r_{j}\left(s\left(\frac{x}{t}\right)\right) \\
\frac{x}{t}=\lambda_{j}\left(\frac{x}{t}\right)
\end{array}\right.
$$

It is constructed tangent with the $j$ right eigenvector. Noting $I_{j}$ a $j$ Riemann invariant, $I_{j}$ is constant along the trajectories of the vector field $r_{j}$ :

$$
\forall W_{n} \quad \nabla I\left(W_{n}\right)^{t} \cdot r_{j}\left(W_{n}\right)=0
$$

Riemann invariants are, with $\left(\tilde{c}_{i}^{\prime}(\hat{\rho})\right)^{2}=Y_{i} P^{\prime}\left(\hat{\rho} Y_{i}\right)+\frac{10}{9} \frac{K_{i}}{\rho_{i}^{\frac{5}{3}}} \cdot \hat{\rho}^{\frac{2}{3}} \quad i=l, r$

$$
\begin{gathered}
I_{1}=\left(Y, u_{n}+\int_{0}^{\rho} \frac{\hat{c}_{l}^{\prime}(\hat{\rho})}{\hat{\rho}} d \hat{\rho}, u_{\tau}, \frac{K}{\rho^{\frac{5}{3}}}\right) \quad I_{2}=\left(\rho Y, u_{n}, u_{\tau}, P(\rho Y)+\frac{2}{3} K\right), \quad I_{3}=\left(u_{n}, u_{\tau}, P(\rho Y)+\frac{2}{3} K, \rho\right) \\
I_{4}=\left(Y, \rho, u_{n}, P(\rho Y)+\frac{2}{3} K\right), \quad I_{5}\left(Y, u_{n}-\int_{0}^{\rho} \frac{\tilde{c}_{r}^{\prime}(\hat{\rho})}{\hat{\rho}} d \hat{\rho}, u_{\tau}, \frac{K}{\rho^{5 / 3}}\right)
\end{gathered}
$$

By the way, we note that both $u_{n}$ and $P+\frac{2}{3} K$ are Riemann invariants through the $2-3-4$ wave. The rarefaction curves are thus given by the following relations :

$$
\begin{gathered}
\mathcal{R}_{1}\left(W_{l}\right)=\left\{\left(Y, \rho, u_{n}, u_{\tau}, K\right), Y=Y_{l}, u_{\tau}=u_{\tau l}, \rho>0, K=\frac{K_{l} \rho^{5 / 3}}{\rho_{l}^{5 / 3}}, u_{n}=u_{n l}+\int_{\rho}^{\rho_{l}} \frac{\tilde{c}_{l}^{\prime}(\hat{\rho})}{\hat{\rho}} d \hat{\rho}\right\} \\
\mathcal{R}_{5}\left(W_{r}\right)=\left\{\left(Y, \rho, u_{n}, u_{\tau}, K\right), Y=Y_{r}, u_{\tau}=u_{\tau r}, \rho>0, K=\frac{K_{r} \rho^{5 / 3}}{\rho_{r}^{5 / 3}}, u_{n}=u_{n r}-\int_{\rho}^{\rho_{r}} \frac{\tilde{c}_{r}^{\prime}(\hat{\rho})}{\hat{\rho}} d \hat{\rho}\right\}
\end{gathered}
$$

- Shock curves are the discontinuous solutions. They must comply with the Rankine-Hugoniot 
jump conditions, noting $\sigma$ the speed of the associated discontinuity:

$$
(\mathrm{R}-\mathrm{H})\left\{\begin{array}{l}
{\left[\rho Y\left(u_{n}-\sigma\right)\right]=0} \\
{\left[\rho\left(u_{n}-\sigma\right)\right]=0} \\
{\left[\rho u_{n}\left(u_{n}-\sigma\right)+P+\frac{2}{3} K\right]=0} \\
{\left[\rho u_{\tau}\left(u_{n}-\sigma\right)\right]=0} \\
{\left[K\left(u_{n}-\sigma\right)+\frac{2}{3} \bar{K} u_{n}\right]=0}
\end{array}\right.
$$

For the nonconservative equation we have an approximate jump relation depending of the choice of the integration's path $\phi(s)$. We refer to [6] for the theory of the nonconservative hyperbolic systems (see also [10], [18]) Here for simplicity we use the straight line's path, in terms of the $\tilde{W}=\left(\rho, Y, u_{n}, u_{\tau}, K\right)$ variables :

$$
\begin{gathered}
\phi\left(s, \tilde{W}_{l}, \tilde{W}_{r}\right)=\tilde{W}_{l}+s\left(\tilde{W}_{r}-\tilde{W}_{l}\right) \\
\int_{\mathbb{R}} K \partial_{x} u d x=\int_{0}^{1} K\left(\phi\left(s, \tilde{W}_{l}, \tilde{W}_{r}\right)\right) \partial_{s} u\left(\phi\left(s, \tilde{W}_{l}, \tilde{W}_{r}\right)\right) d s=\int_{0}^{1}\left(K_{l}+s\left(K_{r}-K_{l}\right)\right)\left(u_{r}-u_{l}\right) d s \\
\int_{\mathbb{R}} K \partial_{x} u d x=\bar{K}[u] \quad \text { with } \quad \bar{K}=\frac{K_{l}+K_{r}}{2}
\end{gathered}
$$

The associated shock curves are :

$$
\begin{gathered}
\mathcal{S}_{1}\left(\tilde{W}_{l}\right)=\left\{\left(\rho, Y, u_{n}, u_{\tau}, K\right), \rho>0, Y=Y_{l}, u_{\tau}=u_{\tau l}, K=\frac{4 \rho-\rho_{l}}{4 \rho_{l}-\rho} K_{l},\right. \\
u_{n}=u_{n l}-\sqrt{\left.\frac{\left.\left(\rho-\rho_{l}\right)\left[\frac{2}{3}\left(K-K_{l}\right)+P-P_{l}\right]\right)}{\rho \rho_{l}}\right\}} \\
\mathcal{S}_{5}\left(\tilde{W}_{r}\right)=\left\{\left(\rho, Y, u_{n}, u_{\tau}, K\right), \rho>0, Y=Y_{r}, u_{\tau}=u_{\tau r}, K=\frac{4 \rho-\rho_{r}}{4 \rho_{r}-\rho} K_{r},\right. \\
\left.u_{n}=u_{n r}+\sqrt{\frac{\left(\rho-\rho_{r}\right)\left[\frac{2}{3}\left(K-K_{r}\right)+\left(P-P_{r}\right)\right]}{\rho \rho_{r}}}\right\}
\end{gathered}
$$

The selection among the solutions, of the curve that admits the right sign is obtained by Lax inequalities. The choice of $\left[u_{n}\right] \leq 0$ will also be justified by the entropy characterization in the following section.

In these solutions we only keep the part of the solution curves where the turbulence is positive, thus we obtain conditions which are exactly similar to the realizability conditions :

$$
\begin{array}{cl}
\tilde{W}_{n} \in \mathcal{S}_{1}\left(\tilde{W}_{l}\right) \quad: \quad & \rho_{l}<\rho<4 \rho_{l} \\
\tilde{W}_{n} \in \mathcal{S}_{5}\left(\tilde{W}_{r}\right) \quad: \quad & \rho_{r}<\rho<4 \rho_{r}
\end{array}
$$


- We emphasize that in the case of a "contact discontinuity", these approximate RankineHugoniot conditions and the rarefaction curves provide the same relations between states on each side of this contact discontinuity, which must be related to the frame of systems of conservative laws (see tests cases in [8]).

$$
\left[u_{n}\right]_{1}^{2}=0 \quad \text { and } \quad\left[\frac{2 K}{3}+P\right]_{1}^{2}=0
$$

Note also that provided $W_{l}$ and $W_{r}$ such that $u_{l}=u_{r}$ and $\left(P+\frac{2 K}{3}\right)_{l}=\left(P+\frac{2 K}{3}\right)_{r}$ then the solution of the one dimensional Riemann problem is an unsteady contact discontinuity traveling with velocity $\sigma=u_{n l}=u_{n r}: W(x, t)=W_{l}$ if $x<\sigma t$ and $W(x, t)=W_{r}$ if $x>\sigma t$

\subsubsection{Scalar resolution of a multidimensional system}

This section is devoted to the computation of an exact solution of (RP). We know that the solution of $(P R)$ is self-similar $W_{n}(x, t)=W^{*}\left(\frac{x}{t}, W_{l}, W_{r}\right)$ and consists in at most four constant states separated by shock waves, (and-or) rarefaction waves and a contact discontinuity [13]. Using Smoller's kind of parameterization [21] of the solution waves, we connect the external states $W_{l}, W_{r}$ to the intermediate ones $W_{1}$ and $W_{2}$. In order to agree with the positivity of $K$, $X_{i}$ describes the following domain :

For a shock : $\left.\left.X_{i} \in\right] \frac{1}{4}, 1\right]$, for a rarefaction wave : $\left.X_{i} \in\right] 1, \infty[$

\begin{tabular}{|l|l|}
\hline From left to 1 state $:$ & From 2 to right state $:$ \\
\hline$\rho_{1}=\frac{\rho_{l}}{X_{1}}$ & $\rho_{2}=\frac{\rho_{r}}{X_{3}}$ \\
$u_{1}=u_{l}+h_{1}\left(X_{1}\right)$ & $u_{2}=u_{r}+h_{3}\left(X_{3}\right)$ \\
$Y_{1}=Y_{l}$ & $Y_{2}=Y_{r}$ \\
$K_{1}=g\left(X_{1}\right) K_{l}$ & $K_{2}=g\left(X_{3}\right) K_{r}$ \\
\hline
\end{tabular}

To connect the states $W_{1}$ and $W_{2}$, we have to solve:

$$
\left\{\begin{array}{lll}
{\left[\frac{2}{3} K+P\right]_{1}^{2}=0} & \Rightarrow F_{1}(X 1, X 3)=0 \\
{[u]_{1}^{2}=0} & \Rightarrow F_{2}(X 1, X 3)=0
\end{array}\right.
$$




$$
\begin{aligned}
& F_{1}(X 1, X 3)=g\left(X_{1}\right) K_{l}+\frac{3}{2}\left(P\left(Y_{l} \frac{\rho_{l}}{X_{1}}\right)-P\left(Y_{r} \frac{\rho_{r}}{X_{3}}\right)\right)-g\left(X_{3}\right) K_{r} \\
& F_{2}(X 1, X 3)=h_{1}\left(X_{1}\right)-h_{3}\left(X_{3}\right)+u_{l}-u_{r} \\
& g(X)= \begin{cases}\left(\frac{1}{X}\right)^{5 / 3} & \text { if } X \geq 1 \\
\frac{4-X}{4 X-1} & \text { if } \frac{1}{4} \leq X \leq 1\end{cases} \\
& h_{1}\left(X_{1}\right)= \begin{cases}-\sqrt{\frac{\left(1-X_{1}\right)\left[\frac{2}{3} K_{l}\left(g\left(X_{1}\right)-1\right)+P\left(Y_{l} \frac{\rho_{l}}{X_{1}}\right)-P\left(Y_{l} \rho_{l}\right)\right]}{\rho_{l}}} & \text { if } \frac{1}{4}<X_{1}<1 \\
\int_{\frac{\rho_{l}}{X_{1}}}^{\rho_{l}} \frac{\tilde{c}_{l}^{\prime}(\hat{\rho})}{\hat{\rho}} d \hat{\rho} & \text { if } X_{1}>1\end{cases} \\
& h_{3}\left(X_{3}\right)= \begin{cases}\sqrt{\frac{\left(1-X_{3}\right)\left[\frac{2}{3} K_{r}\left(g\left(X_{3}\right)-1\right)+P\left(Y_{r} \frac{\rho_{r}}{X_{3}}\right)-P\left(Y_{r} \rho_{r}\right)\right]}{\rho_{r}}} & \text { if } \frac{1}{4}<X_{3}<1 \\
-\int_{\frac{\rho_{r}}{X_{3}}}^{\rho_{r}} \frac{\tilde{c}_{r}^{\prime}(\hat{\rho})}{\hat{\rho}} d \rho & \text { if } X_{3}>1\end{cases}
\end{aligned}
$$

To V.N.L. fields may correspond an approximate shock solution or an exact rarefaction wave.

Proposition 3 Assume that approximate jump conditions (47) hold. Then the one dimensional Riemann problem associated with the nonconservative convective system $\left(\mathcal{S}_{c}\right)$ has a unique entropy-consistent solution with no vacuum occurrence provided that:

$$
u_{n r}-u_{n l}<Z_{l}+Z_{r} \quad \text { with } Z_{i}=\int_{0}^{\rho_{i}} \frac{\tilde{c}_{i}^{\prime}(\hat{\rho})}{\hat{\rho}} d \hat{\rho}
$$

Sketch of proof :

By the strict monotonicity of $F_{2}\left(X_{1}, X_{3}\right)$, which is a growing function of $X_{1}$, we deduce that if $h_{3}\left(X_{3}\right)+u_{r}-u_{l}>Z_{l}$, there exists $X_{1}=\varphi\left(X_{3}\right)$. The $\varphi$ function is a strictly nongrowing function of $X_{3}$; thus $X_{1}\left(X_{3}\right)$ is unique. Moreover, $F_{1}\left(X_{1}\left(X_{3}\right), X_{3}\right)$ is a strictly growing function of $X_{3}$, 
and, as $-h_{3}\left(X_{3}\right)<Z_{r}$ we conclude by this computation that we get a unique couple $\left(X_{1}, X_{3}\right)$ if and only if $u_{n r}-u_{n l}<Z_{l}+Z_{r}$.

This result is exact if the connection between states is a rarefaction wave or a contact discontinuity. If to V.N.L. fields corresponds a shock solution, we have to assume that the jump's amplitude remains weak. The positivity of $\rho, K, Y$ and $1-Y$ is checked by the parameterization (55), (58) and the realizability (53).

In regular waves, since Riemann invariants are preserved, the following behavior of the turbulent Mach number holds:

$$
M_{\text {turb }} \approx\left(\frac{K}{P}\right)^{\frac{1}{2}} \approx(\rho)^{\frac{5-3 \gamma}{6}}
$$

where the exponent is usually positive since $\gamma<\frac{5}{3}$ in most practical applications. Thus, this number is decreasing in low density regions.

In the following section, we assess the choice of density variations through shock waves, on the basis of an entropy inequality.

\section{Entropy functions and uniqueness of the solution}

\subsection{Entropy functions}

For the conservative system $\left(\mathcal{S}_{c}\right)$, a convex function $\varphi: \Omega \rightarrow \mathbb{R}$ is an entropy if there exists a flux function $f_{\varphi}: \Omega \rightarrow \mathbb{R}$ so that $:$

$$
\left\{\begin{array}{lc}
\partial_{t} \mathcal{U}+\nabla(G(\mathcal{U}))=0 & \left(\mathcal{S}_{c}\right) \\
(\nabla \varphi(\mathcal{U}))^{t} \frac{d G(\mathcal{U})}{d \mathcal{U}}=\left(\nabla f_{\varphi}(\mathcal{U})\right)^{t} & \forall \mathcal{U} \in \Omega
\end{array}\right.
$$

The piecewise $\mathcal{C}_{1}$ function $\mathcal{U}$ is an entropy solution of $\left(\mathcal{S}_{c}\right)$ if $\mathcal{U}$ is a classical solution of $\left(\mathcal{S}_{c}\right)$ where $\mathcal{U}$ is $\mathcal{C}_{1}$ and satisfies the Rankine-Hugoniot conditions in the discontinuities, and further more satisfies for each entropy function $\varphi$ the jump inequality :

$$
\sigma[\varphi(\mathcal{U})] \geq\left[f_{\varphi}(\mathcal{U})\right]
$$

So, we have to find a new variable $\varphi$, that is a combination of $K$ and the other variables, such that its evolution equation would be conservative. As we can see in the following proposition we have a conservative formulation of the convective part our $(\mathcal{S})$ system. 


\section{Proposition 4}

$\varphi=\frac{K}{\rho^{2 / 3}}$ is an entropy function of $(\mathcal{S}): \quad \partial_{t}\left(\frac{K}{\rho^{2 / 3}}\right)+\nabla\left(\frac{K}{\rho^{2 / 3}} U\right)=0$

$$
\begin{aligned}
\partial_{t}\left(\frac{K}{\rho^{2 / 3}}\right) & =\frac{1}{\rho^{2 / 3}} \partial_{t} K-\frac{2}{3} \frac{K}{\rho^{5 / 3}} \partial_{t} \rho \\
& =-\frac{1}{\rho^{2 / 3}} \nabla(K U)-\frac{2 K}{3 \rho^{2 / 3}} \nabla U+\frac{2 K}{3 \rho^{5 / 3}} \nabla(\rho U)=-\nabla\left(\frac{K}{\rho^{2 / 3}} U\right)
\end{aligned}
$$

We note this entropy-entropy flux pair $\mathcal{F}=\frac{K}{\rho^{2 / 3}}, f_{\mathcal{F}}=\frac{K u}{\rho^{2 / 3}}$.

$(\mathcal{S})$ is conservative in $\mathcal{U}=\left(\rho, \rho Y, \rho U, \frac{K}{\rho^{2 / 3}}\right): \quad\left(\mathcal{S}_{c}\right) \quad \partial_{t} \mathcal{U}+\nabla(G(\mathcal{U}))=0$

$$
\left(\mathcal{S}_{c}\right)\left\{\begin{array}{l}
\partial_{t} \rho+\nabla(\rho U)=0 \\
\partial_{t}(\rho Y)+\nabla(\rho Y U)=0 \\
\partial_{t}(\rho U)+\nabla\left(\rho U^{2}+\frac{2}{3} K+P \overline{\bar{T}}\right)=0 \\
\partial_{t}\left(\frac{K}{\rho^{2 / 3}}\right)+\nabla\left(\frac{K}{\rho^{2 / 3}} u\right)=0
\end{array}\right.
$$

Theorem 1 The generic formulation of the $\varphi$ entropies of $(\mathcal{S})$ is :

$$
\varphi=C_{1}\left(\frac{\rho U^{2}}{2}+\rho \int \frac{P(\rho Y)}{\rho^{2}} d \rho+K\right)+C_{2} \rho+C_{3} \rho Y+C_{4} \rho U+C_{5} \frac{K}{\rho^{2 / 3}}+C_{6}
$$

$\varphi$ is a combination of the conservative variables of $\left(\mathcal{S}_{c}\right)$.

Further more, we have found a new entropy $\mathcal{E}$, that is the total energy of the mean flow.

$$
\mathcal{E}=\frac{\rho U^{2}}{2}+K+\rho \int \frac{P(\rho Y)}{\rho^{2}} d \rho
$$

Proof Hence, to identify all the entropies $\varphi$ of $(\mathcal{S})$, we use the propriety applied to $\left(\mathcal{S}_{c}\right)$ that $D^{2} \varphi \frac{d G}{d \mathcal{U}}$ must be a symmetrical matrix. See $[7]$ for details.

Proposition 5 The associated entropy flux function $f_{\mathcal{E}}$ of $\mathcal{E}$ is :

$$
f_{\mathcal{E}}=\frac{\rho U^{3}}{2}+\frac{5}{3} K U+\rho U \int \frac{P(\rho Y)}{\rho^{2}} d \rho+U P
$$




$$
\begin{aligned}
\partial_{t}\left(\rho \int \frac{P}{\rho^{2}} d \rho\right) & =\int \frac{P}{\rho^{2}} d \rho \partial_{t} \rho+\frac{P}{\rho} \partial_{t} \rho+\rho \int \frac{\partial P}{\partial Y} \frac{1}{\rho^{2}} d \rho \partial_{t} Y \\
& =-\nabla(\rho u)\left(\int \frac{P}{\rho^{2}} d \rho+\frac{P}{\rho}\right)-\nabla(\rho Y u) \int \frac{\partial P}{\partial Y} \frac{1}{\rho^{2}} d \rho+Y \nabla(\rho u) \int \frac{\partial P}{\partial Y} \frac{1}{\rho^{2}} d \rho \\
& =-\nabla\left(\rho u \int \frac{P}{\rho^{2}} d \rho\right)-P \nabla u-Y \nabla(\rho u) \int \frac{\partial P}{\partial Y} \frac{1}{\rho^{2}} d \rho+\nabla(\rho u) Y \int \frac{\partial P}{\partial Y} \frac{1}{\rho^{2}} d \rho \\
& =-\nabla\left(\rho u \int \frac{P}{\rho^{2}} d \rho\right)-P \nabla u \\
\partial_{t}\left(\frac{\rho U^{2}}{2}+K\right) & =-U \nabla\left(\rho U^{2}+\frac{2}{3} K+P \overline{\bar{I}}\right)+\frac{U^{2}}{2} \nabla(\rho U)-\nabla(K U)-\frac{2}{3} K \nabla U \\
\partial_{t}\left(\frac{\rho U^{2}}{2}\right. & \left.+K+\rho \int \frac{P}{\rho^{2}} d \rho\right)+\nabla\left(\frac{\rho U^{3}}{2}+\frac{5}{3} K U+\rho U \int \frac{P(\rho Y)}{\rho^{2}} d \rho+P U\right)=0
\end{aligned}
$$

Remark : It is not possible to symmetrize the system with the variable $\frac{\partial \mathcal{E}}{\partial W}$. One should thus consider other variables for numerical purposes involving Petrov-Galerkin approach (see [16] for instance).

\subsection{A unique physical entropy}

Thanks to the vanishing viscosity method, we can show that the mathematical entropy $\mathcal{E}$ is consistant with the viscous terms of the convective-diffusive system $(\mathcal{S})$. Then, for our system, we proof the equivalence between the Lax inequalities and the compressive shock. At last, we show that the growing on shocks of the entropy $\mathcal{F}$ implies incompressive shock and then, $\mathcal{F}$ has no physical sense.

\subsection{1 $\mathcal{E}$ is a physical entropy}

Keeping in mind the second principle of thermodynamics, a $\varphi$ convex entropy is growing on a physical shock.

$$
\sigma[\varphi(\mathcal{U})] \geq\left[f_{\varphi}(\mathcal{U})\right]
$$

We will show that we have this inequality for the entropy-entropy flux pair $\left(\mathcal{E}, f_{\mathcal{E}}\right)$.

Theorem 2 The entropy-entropy flux pair $\left(\mathcal{E}, f_{\mathcal{E}}\right)$ is consistent with the viscous terms of $(\mathcal{S})$ 


\section{Proof :}

Let us consider the $(\mathcal{S})$ system written as follow, in 1D frame :

$$
\begin{gathered}
\partial_{t} W+A(W) \partial_{x} W=\partial_{x}\left(C(W) \partial_{x} W\right)+D\left(W, \partial_{x} W\right)-E(W) \\
W=(\rho, \rho Y, \rho u, K)^{t} \quad D\left(W, \partial_{x} W\right)=\left(0,0,0, \frac{4}{3} \mu_{t}\left(\partial_{x} u\right)^{2}\right)^{t} \quad E(w)=(0,0,0, \rho \varepsilon)^{t} \\
A=\left(\begin{array}{cccc}
0 & 0 & 1 & 0 \\
-Y u & u & Y & 0 \\
-u^{2} & P^{\prime}(\rho Y) & 2 u & \frac{2}{3} \\
-\frac{5}{3} K u & 0 & \frac{5}{3} \frac{K}{\rho} & u
\end{array}\right) \quad C=\left(\begin{array}{cccc}
0 & 0 & 0 & 0 \\
0 & 0 & 0 & 0 \\
-\mu_{\mathrm{eff}} \frac{4}{3} \frac{u}{\rho} & 0 & \mu_{\mathrm{eff}} \frac{4}{3} \frac{1}{\rho} & 0 \\
-\eta \frac{K}{\rho^{2}} & 0 & 0 & \frac{\eta}{\rho}
\end{array}\right) \\
\mathcal{E}(\mathcal{W})=\frac{\rho u^{2}}{2}+K+\rho \int \frac{P(\rho Y)}{\rho^{2}} d \rho \\
f_{\mathcal{E}}(W)=\frac{\rho u^{3}}{2}+\frac{5}{3} K u+\rho u \int \frac{P(\rho Y)}{\rho^{2}} d \rho+U P
\end{gathered}
$$

By the following computation, we obtain the equation verified by the entropy :

$$
\begin{aligned}
\left(\frac{\partial \mathcal{E}(W)}{\partial W}\right)^{t}\left(\partial_{t} W+A(W) \partial_{x} W\right)=\left(\frac{\partial \mathcal{E}(W)}{\partial W}\right)^{t}\left(\partial_{x}\left(C(W) \partial_{x} W\right)+D\left(W, \partial_{x} W\right)-E(W)\right) & \\
\partial_{t}(\mathcal{E}(W))+\partial_{x}\left(f_{\mathcal{E}}(W)\right) & =\left(-u^{2}, \frac{P^{\prime}(\rho Y)}{\gamma-1}, u, 1\right)\left(0,0, \frac{4}{3} \partial_{x}\left(\mu_{e f f} \partial_{x} u\right), \frac{4}{3} \mu_{t}\left(\partial_{x} u\right)^{2}+\partial_{x}\left(\eta \partial_{x}\left(\frac{K}{\rho}\right)-\rho \varepsilon\right)^{t}\right. \\
& =\frac{4}{3}\left(u \partial_{x}\left(\mu_{e f f} \partial_{x} u\right)+\mu_{t}\left(\partial_{x} u\right)^{2}\right)+\partial_{x}\left(\eta \partial_{x}\left(\frac{K}{\rho}\right)\right)-\rho \varepsilon \\
& =\frac{4}{3}\left(\mu_{e f f} \partial_{x}\left(u \partial_{x} u\right)+\left(\mu_{t}-\mu_{e f f}\right)\left(\partial_{x} u\right)^{2}\right)+\partial_{x}\left(\eta \partial_{x}\left(\frac{K}{\rho}\right)\right)-\rho \varepsilon \\
& =\partial_{x}\left(\frac{4}{3} \mu_{e f f} u \partial_{x} u+\eta \partial_{x}\left(\frac{K}{\rho}\right)\right)-\frac{4}{3} \mu_{l a m}\left(\partial_{x} u\right)^{2}-\rho \varepsilon
\end{aligned}
$$

Using traveling waves $W(x, t)=W(\xi)=W(x-\sigma t)$ we have :

$$
-\sigma \mathcal{E}^{\prime}(W)(\xi)+f_{\mathcal{E}}^{\prime}(W)(\xi)=\left(\frac{4}{3} \mu_{e f f} u \partial_{x} u+\eta \partial_{x}\left(\frac{K}{\rho}\right)\right)^{\prime}(\xi)-\frac{4}{3} \mu_{l a m}\left(\partial_{x} u\right)^{2}(\xi)-\rho \varepsilon(\xi)
$$

Integrating between left and right states, with $\lim _{\xi \rightarrow-\infty} W=W_{l}$ and $\lim _{\xi \rightarrow+\infty} W=W_{r}$ :

$$
-\sigma[\mathcal{E}(W)]+\left[f_{\mathcal{E}}(W)\right]=\left[\frac{4}{3} \mu_{e f f} u \partial_{x} u+\eta \partial_{x}\left(\frac{K}{\rho}\right)\right]-\frac{4}{3} \mu_{l a m} \int_{\mathbb{R}}\left(\partial_{x} u\right)^{2}(\xi) d \xi-\int_{\mathbb{R}} \rho \varepsilon(\xi) d \xi
$$

As $\lim _{-\infty} \partial_{x} u=\lim _{+\infty} \partial_{x} u=0$ and $\lim _{-\infty} \partial_{x}\left(\frac{K}{\rho}\right)=\lim _{+\infty} \partial_{x}\left(\frac{K}{\rho}\right)=0$ :

$$
-\sigma[\mathcal{E}(W)]+\left[f_{\mathcal{E}}(W)\right]=-\frac{4}{3} \mu_{l a m} \int_{\mathbb{R}}\left(\partial_{x} u\right)^{2} d \xi-\int_{\mathbb{R}} \rho \varepsilon(\xi) d \xi \leq 0
$$


Assuming that $\mu_{\text {lam }}$ and also that the turbulent dissipation remains positives, we conclude that

$$
\sigma[\mathcal{E}(W)] \geq\left[f_{\mathcal{E}}(W)\right]
$$

We should notice that the entropic dissipation of our system is very weak. It just depends on the laminar viscosity, which is quite negligible compared with the turbulent viscosity $\mu_{t}$ (see [12], [15] for somewhat similar entropic considerations.). We should remark too, that both contributions of (79) $\mu_{\text {lam }}\left(\partial_{x} u\right)^{2}$ and $-\rho \varepsilon$ are proportional to $\mu_{\text {lam }}$. Even more, their sum exactly corresponds to the average of the instantaneous dissipation, so this sum disappears as soon as $\mu_{\text {lam }}$ vanishes. Thus we have obtained a physically relevant entropy inequality. Straightforward though tedious algebra manipulations enable to conclude that $\left[u_{n}\right] \leq 0$ using entropy inequality and inserting approximate jump conditions inside.

\subsubsection{The $\mathcal{F}$ entropy has no physical sense}

With same considerations on the entropy $\mathcal{F}$, we can't easily conclude on the sign of the entropic dissipation.

$$
-\sigma[\mathcal{F}(W)]+\left[f_{\mathcal{F}}(W)\right]=\int_{\mathbb{R}} \frac{1}{\rho^{2 / 3}}\left(\partial_{x}\left(\eta \partial_{x}\left(\frac{K}{\rho^{2 / 3}}\right)+\frac{4}{3} \mu_{t}\left(\partial_{x} u\right)^{2}\right)(\xi) d \xi-\int_{\mathbb{R}} \rho^{1 / 3} \varepsilon(\xi) d \xi\right.
$$

So, we use others arguments to come to the conclusion that the no entropy inequality arises from the latter.

- Lax inequalities and compressive shock

We demonstrate the equivalence, for our system, between the Lax inequalities (which select the entropic solution) and the growing of density on shock curves (in the positive travel sense).

We recall the Lax inequalities on a 1-shock curve between the states 1 and 2 , setting $v_{i}=u_{i}-\sigma:$

$$
\left\{\begin{array} { l } 
{ \sigma < u _ { 2 } } \\
{ u _ { 2 } - c _ { 2 } ^ { \prime } < \sigma < u _ { 1 } - c _ { 1 } ^ { \prime } }
\end{array} \Leftrightarrow \left\{\begin{array} { l } 
{ v _ { 2 } < c _ { 2 } ^ { \prime } , v _ { 2 } > 0 } \\
{ v _ { 1 } > c _ { 1 } ^ { \prime } }
\end{array} \Leftrightarrow \left\{\begin{array}{l}
c_{2}^{\prime 2}>v_{2}^{2} \\
v_{1}^{2}>c_{1}^{\prime 2}
\end{array}\right.\right.\right.
$$

Theorem 3 On a 1-shock curve we have the equivalence:

$$
\left\{\begin{array}{c}
c_{2}^{\prime 2}>v_{2}^{2} \\
v_{1}^{2}>c_{1}^{\prime 2}
\end{array} \Leftrightarrow \rho_{2}>\rho_{1}\right.
$$


We have the opposite direction in a 4-shock curve : $\rho_{2}<\rho_{1}$

- We begin with the implication on a 1-shock curve : $\left\{\begin{array}{l}c_{2}^{\prime 2}>v_{2}^{2} \\ v_{1}^{2}>c_{1}^{\prime 2}\end{array} \Rightarrow \rho_{2}>\rho_{1}\right.$

By a reductio ad absurdum, we suppose $\rho_{1}>\rho_{2}$ :

$$
\begin{gathered}
c_{2}^{\prime}>v_{2}^{2} \text { and with the jump relation }[\rho v]=0 \Rightarrow v_{2}^{2}=\frac{\rho_{1}^{2}}{\rho_{2}^{2}} v_{1}^{2} \\
v_{1}^{2}>c_{1}^{\prime 2} \Rightarrow c_{2}^{\prime}>\frac{\rho_{1}^{2}}{\rho_{2}^{2}} c_{1}^{2} \\
c_{2}^{\prime 2}=\left(\frac{\partial P}{\partial \rho}\right)\left(\rho_{2}\right)+\frac{10 K_{2}}{9 \rho_{2}} \\
\left.\left(\frac{\partial P}{\partial \rho}\right)\left(\rho_{2}\right)+\frac{10 K_{2}}{9 \rho_{2}}>\frac{\rho_{1}^{2}}{\rho_{2}^{2}}\left(\frac{\partial P}{\partial \rho}\right)\left(\rho_{1}\right)+\frac{10 K_{1}}{9 \rho_{1}}\right) \\
\left(\frac{\partial P}{\partial \rho}\right)\left(\rho_{2}\right)\left[1-\frac{\rho_{1}^{2}\left(\frac{\partial P}{\partial \rho}\right)\left(\rho_{1}\right)}{\rho_{2}^{2}\left(\frac{\partial P}{\partial \rho}\right)\left(\rho_{2}\right)}\right]>-\frac{10 K_{2}}{9 \rho_{2}}\left[1-\frac{\rho_{1} K_{1}}{\rho_{2} K_{2}}\right]
\end{gathered}
$$

We have the negativity of the first member because of the $P$ growth. But, the second member is positive by realizability :

$$
\begin{gathered}
K_{1}-K_{2}=\frac{5\left(\rho_{1}-\rho_{2}\right)}{4 \rho_{2}-\rho_{1}} K_{1} \\
4 \rho_{2}-\rho_{1}>0 \Rightarrow K_{1}>K_{2} \Rightarrow 1-\frac{\rho_{1} K_{1}}{\rho_{2} K_{2}}>0
\end{gathered}
$$

By a same reasoning we conclude for a 4 -shock curve that :

$$
\left\{\begin{array}{c}
v_{2}^{2}>c_{2}^{\prime 2} \\
v_{1}^{2}<c_{1}^{\prime 2}
\end{array} \Rightarrow \rho_{1}>\rho_{2}\right.
$$

- Then we show the reverse : For a 1 -shock $\rho_{2}>\rho_{1} \Rightarrow c_{2}^{\prime 2}>v_{2}^{2} \quad v_{1}^{2}>c_{1}^{\prime 2}$

$$
\begin{aligned}
& \sigma_{1}=u_{1}-\sqrt{\frac{\rho_{2}\left(\frac{2}{3}\left(K_{2}-K_{1}\right)+P_{2}-P_{1}\right)}{\rho_{1}\left(\rho_{2}-\rho_{1}\right)}} \\
& \sigma_{1}=u_{2}-\sqrt{\frac{\rho_{1}\left(\frac{2}{3}\left(K_{2}-K_{1}\right)+P_{2}-P_{1}\right)}{\rho_{2}\left(\rho_{2}-\rho_{1}\right)}}
\end{aligned}
$$




$$
\frac{\rho_{1}}{\rho_{2}} c_{1}^{\prime 2}<\frac{\left(\frac{2}{3}\left(K_{2}-K_{1}\right)+P_{2}-P_{1}\right)}{\rho_{2}\left(\rho_{2}-\rho_{1}\right)}<\frac{\rho_{2}}{\rho_{1}} c_{2}^{\prime 2}
$$

We have to demonstrate the two inequalities:

$$
\begin{gathered}
\rho_{2} c_{2}^{\prime 2}\left(\rho_{2}-\rho_{1}\right)-\frac{2}{3} \rho_{1}\left(K_{2}-K_{1}\right)-\rho_{1}\left(P_{2}-P_{1}\right)>0 \\
\rho_{1}\left(c_{1}^{\prime}\right)^{2}\left(\rho_{2}-\rho_{1}\right)-\frac{2}{3} \rho_{2}\left(K_{2}-K_{1}\right)-\rho_{2}\left(P_{2}-P_{1}\right)<0
\end{gathered}
$$

We use the propriety of $Y$ constant on shock curves, so $c^{2}=\frac{\partial P}{\partial \rho}+\frac{10 K}{9 \rho}$

We show separately :

$$
\begin{gathered}
\text { (89) }\left\{\begin{array}{cc}
(89-1) & \rho_{2}\left(\rho_{2}-\rho_{1}\right) \frac{\partial P}{\partial \rho}\left(\rho_{2}\right)-\rho_{1}\left(P_{2}-P_{1}\right)>0 \\
(89-2) & \frac{10}{9} K_{2}\left(\rho_{2}-\rho_{1}\right)-\frac{2}{3} \rho_{1}\left(K_{2}-K_{1}\right)>0
\end{array}\right. \\
(90) \begin{cases}(90-1) & \rho_{1}\left(\rho_{2}-\rho_{1}\right)\left(\frac{\partial P}{\partial \rho}\right)\left(\rho_{1}\right)-\rho_{2}\left(P_{2}-P_{1}\right)<0 \\
(90-2) & \frac{10}{9} K_{1}\left(\rho_{2}-\rho_{1}\right)-\frac{2}{3} \rho_{2}\left(K_{2}-K_{1}\right)<0\end{cases}
\end{gathered}
$$

Setting $f_{1}\left(\rho_{1}, \rho\right)=\rho\left(\rho-\rho_{1}\right) \frac{\partial P}{\partial \rho}(\rho)-\rho_{1}\left(P_{2}-P_{1}\right)$, then $f_{1}\left(\rho_{1}, \rho_{1}\right)=0$

$\frac{\partial f_{1}}{\partial \rho}\left(\rho_{1}, \rho\right)=\left(\rho-\rho_{1}\right) \underbrace{\left(2 \frac{\partial P}{\partial \rho}(\rho)+\rho \frac{\partial^{2} P}{\partial \rho^{2}}\right)}_{>0} \Rightarrow\left(\rho-\rho_{1}\right) \frac{\partial f_{1}}{\partial \rho}\left(\rho_{1}, \rho\right)>0$

And for $\rho_{2}>\rho_{1}, f_{1}$ is growing, so is positive on $\rho_{2}$. With $\rho_{2}>\rho_{1}$ we get $(89-1)$.

It is the same for $(90-1)$

$$
\begin{gathered}
K_{1}-K_{2}=\frac{5\left(\rho_{1}-\rho_{2}\right)}{4 \rho_{2}-\rho_{1}} K_{1} \Rightarrow K_{2}=\frac{\left(4 \rho_{2}-\rho_{1}\right) K_{1}}{4 \rho_{1}-\rho_{2}} \\
\frac{10}{9} K_{2}\left(\rho_{2}-\rho_{1}\right)-\frac{2}{3} \rho_{1}\left(K_{2}-K_{1}\right)=\frac{10\left(\rho_{2}-\rho_{1}\right)^{2} K_{1}}{9\left(4 \rho_{2}-\rho_{1}\right)}>0
\end{gathered}
$$

It is the same for $(90-2)$, so we conclude on the equivalence between Lax inequalities and compressive shock.

- The shock growing of the entropy $\mathcal{F}$ implies incompressive shock

\section{Theorem 4}

The shock growing of the entropy $\mathcal{F}=\frac{K}{\rho^{\frac{2}{3}}}$ implies incompressive shock. So $\mathcal{F}$ has no physical sense. 


$$
\begin{aligned}
\frac{K_{2} v_{2}}{\rho_{2}^{2 / 3}}-\frac{K_{1} v_{1}}{\rho_{1}^{2 / 3}} & =K_{1}\left[\frac{K_{2}}{K_{1}} \frac{v_{2}}{\rho_{2}^{2 / 3}}-\frac{v_{1}}{\rho_{1}^{2 / 3}}\right] \\
& =\frac{K_{1}}{\rho_{1}^{2 / 3}}\left[\frac{K_{2} v_{2}^{5 / 3}}{K_{1} v_{1}^{2 / 3}}-v_{1}\right]
\end{aligned}
$$

Using the fact that $K_{2}=K_{1} \frac{4 v_{1}-v_{2}}{4 v_{2}-v_{1}}$ :

$$
\frac{K_{2} v_{2}}{\rho_{2}^{2 / 3}}-\frac{K_{1} v_{1}}{\rho_{1}^{2}}=\frac{K_{1}}{\rho_{1}^{2 / 3}}\left[\frac{\left(4 v_{1}-v_{2}\right) v_{2}^{5 / 3}-v_{1}^{5 / 3}\left(4 v_{2}-v_{1}\right)}{v_{1}^{2 / 3}\left(4 v_{2}-v_{1}\right)}\right]
$$

Setting $x=\frac{v_{2}}{v_{1}}$ :

$$
\left[\frac{K v}{\rho \frac{2}{3}}\right]=\frac{K_{1} v_{1}^{2}}{\rho_{1}^{2 / 3}\left(4 v_{2}-v_{1}\right)}\left(-x^{8 / 3}+4 x^{5 / 3}-4 x+1\right)
$$

On a 1 -shock curve $v_{1}>0, v_{2}>0$ and by realizability $4 v_{2}-v_{1}>0$, so, we are interested by the variations on $[0, \infty[$ of the $f$ function :

$$
f(x)=-x^{8 / 3}+4 x^{5 / 3}-4 x+1
$$

$f$ is a nongrowing function, positive on $[0,1]$ and negative on $[1, \infty]$ thus :

$$
\left[\frac{K v}{\rho^{2 / 3}}\right] \text { has the sign of }[-v]
$$

$$
\begin{aligned}
& \text { And }\left[\frac{K v}{\rho^{2 / 3}}\right]<0 \Leftrightarrow \sigma\left[\frac{K}{\rho^{2 / 3}}\right]>\left[\frac{K u}{\rho^{2 / 3}}\right] \text { and }-[v]<0 \Leftrightarrow[\rho]<0 \\
& \sigma\left[\frac{K}{\rho^{2 / 3}}\right]>\left[\frac{K u}{\rho^{2 / 3}}\right] \Leftrightarrow[\rho]<0
\end{aligned}
$$

To conclude, we note that $\mathcal{E}$ is growing on 1-shock curve, like $\rho$, whereas the second mathematical entropy $\mathcal{F}$ does not. Hence $\mathcal{E}=\frac{\rho U^{2}}{2}+K+\rho \int \frac{P(\rho Y)}{\rho^{2}} d \rho$ is the unique physically relevant entropy of our system.

When focusing on the standard $K-\varepsilon$ model, we emphasize that Coquel and Berthon recently proposed ([1], [2]) the use of this "physical entropy" to develop convenient numerical schemes for nonconservative integration systems.

\section{Exact Riemann solution on a shock tube problem}

In this example we give the exact Riemann solution of the following shock test case. The initial states are $\left(\rho_{l}, Y_{l}, u_{l}, K_{l}\right)=(1,0.1,10,1000)$ and $\left(\rho_{r}, Y_{r}, u_{r}, K_{r}\right)=(1,0.9,10,1000)$ with 
the pressure law $P=c(\rho Y)^{5 / 3}$ and $P_{l}=100000 P a$. The solution presented (fig.1) is the projection in $(x, t)$ frame of the exact Riemann solution. The intermediate states obtained by the exact Riemann solver are $\left(\rho_{1}, Y_{1}, u_{1}, K_{1}\right)=(1.965,0.1,-950,843995)$ and $\left(\rho_{2}, Y_{2}, u_{2}, K_{2}\right)=$ $(0.668,0.9,-950,2987.79)$. This solution depends of the approximate jump relation chosen $(50)$. In part II we will present the solutions obtained by means of Godunov scheme and a comparison with some approximate Godunov schemes. We can notice the creation of turbulence on shock and the weak loss of turbulence in the rarefaction wave.
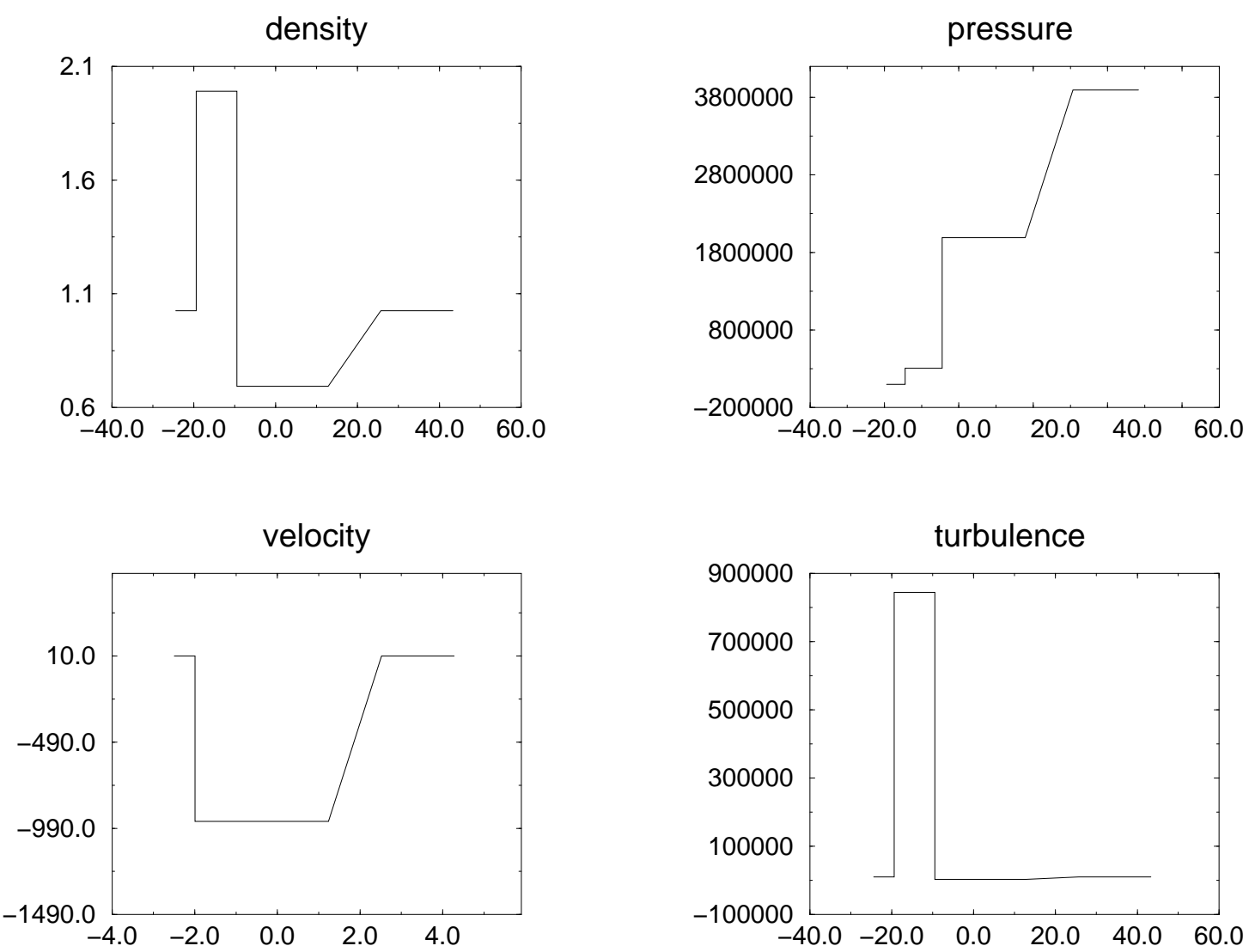

Figure 1: Density, pressure, velocity and turbulence profiles at $\mathrm{t}=0.01 \mathrm{~s}$

\section{Conclusion}

This paper was devoted to the solution of the one dimensional Riemann problem associated with the convective part of a model describing a turbulent multicomponent flow. This was achieved thanks to a physically relevant entropy inequality, which enables to select the unique entropic solution in shock curves, provided some approximate jump conditions hold. The exhibited solution fulfills the realizability requirements, both through rarefaction waves and approximate 
shock curves.

A similar work has been reported when investigating the convective part of the K-epsilon model focusing on compressible flows ([12]), or when dealing with second-moment compressible closures([4]). The whole shows that these models arising from statistical approach of turbulence contain two distinct pressure fields. In all cases, the solution of the Riemann problem requires analysis of a coupled set of four equations (the remaining components -if meaningful- are simply obtained by deduction afterwards), which eventually results in solving a non-linear set of two equations with two unknowns, which can be rather easily done using some Newton algorithm. The ratio of these two pressures represents the square of what is usually called the turbulent Mach number by workers in the turbulent community. Though it is often assumed that this number is negligible in practice, it appears that this hypothesis no longer holds when approaching the wall boundaries, or in shear wakes. As a result, rough application of Euler type schemes to the frame of these complex 'two-pressure' models may generate strong oscillations close to wall boundaries, or in strong rarefaction waves.

As a straightforward consequence of the present approach, Godunov type solvers may be constructed and approximate Riemann solvers may be exhibited, the solutions of which may be compared with exact solution of the Riemann problem. This is achieved in a companion paper [8].

\section{References}

[1] Berthon, C., (1999) Contribution à l'analyse numérique des équations de Navier-Stokes compressibles à deux entropies spécifiques. Applications à la turbulence compressible, $\mathrm{PhD}$ Thesis, University of Paris VI, Paris, France.

[2] Berthon, C., Coquel, F., Convection diffusion system with first and second order in nonconservation form, in preparation.

[3] Baldwin, B. S., Barth, T. J. (1990) A one equation turbulence model for high Reynolds number wall-bounded flows., NASA tec. memorandum $n^{\circ} 102847$. 
[4] Brun, G., Herard, J.M., Jeandel, D., Uhlmann,M. (2000) An approximate Roe-type Riemann solver for a class of realisable second-moment closures, to appear in the International Journal of Computational Fluid Dynamics.

[5] Combe, L.(1997) Simulation mumérique d'écoulements gaz-particules sur maillage non structuré, PhD Thesis, Institut National Polytechnique de Toulouse, France (27/10/1997) . Also as E.D.F report HE-41/97/042/A, Electricite de France, Division Recherche et Developpement, Departement MFTT, 6 quai Watier, 78400 Chatou, France.

[6] Dal Maso, G., Le Floch, P. and Murat, F. (1995) Definition and weak stability of nonconservative products, J. Math. Pures et Appliquées, Vol. $74 n^{\circ} 6$ pp.483-548.

[7] Declercq-Xeuxet, E. (23/06/1999) Comparaison de solveurs numériques pour le traitement de la turbulence bifluide, PhD Thesis, Univ. of Evry, Evry France.

[8] Declercq, E., Forestier, A., Hérard, J.M., Louis, X. and Poissant, G. (2000) Comparison of numerical solvers for a multicomponent turbulent flow, Part 2 submitted to the International Journal of Computational Fluid Dynamics.

[9] Delhaye, J.M.(1968) Equations fondamentales des écoulements diphasiques, Part. 1 et 2 CEA-R-3429, C.E.N. 17, rue des Martyrs, 38054 Grenoble, France.

[10] De Vuyst, F. (19/12/1994) Schémas non conservatifs et schémas cinétiques pour la simulation numérique d'écoulements hypersoniques non visqueux en déséquilibre thermochimique, PhD Thesis, univ. Paris VI., Paris, France.

[11] Favre, A. (dec 1965) Équations des gaz turbulents compressibles, II- Méthode des vitesses moyennes; méthode des vitesses macroscopiques pondérées par la masse volumique, Journal de Mécanique, Vol.4, No.4.

[12] Forestier, A., Hérard, J.M. and Louis, X. (1997) Solveur de type Godunov pour simuler les écoulements turbulents compressibles, C. R. Acad. Sci. Paris, t. 324, Série I, pp.919-926.

[13] Godlewski, E., and Raviart, P.A. (1996) Numerical Approximation of Hyperbolic Systems of Conservation Laws, Applied Mathematical Sciences 118, ED. Springer.

[14] Godunov, S. K. (1957) A finite difference method for the numerical computation of discontinous solutions of the equations of fluid dynamics., Math. Sb. 47, pp.357-393. 
[15] Herard, J.M., Forestier, A. and Louis, X. (1994) A Non Strictly Hyperbolic System To Describe Compressible Turbulence, report E.D.F/D.E.R. HE-41/94/11A, 6 quai Watier 78400 Chatou, France.

[16] Jansen, K., Johan, Z., Hugues T.J.R. (1993) Implementation of a one equation turbulence model within a stabilized finite element formulation of a symmetric advective diffusive system., CMAME vol. 105, pp.405-433.

[17] Kumbaro, A. (10/12/1992) Modélisation, analyse mathématique et numérique des modèles bi-fluides d'écoulement diphasique., PhD Thesis, univ. Paris XI Orsay, France.

[18] Louis, X. (06/07/1995) Modélisation Numérique de la turbulence compressible, PhD Thesis, univ. Paris VI, Paris, France.

[19] Sainsaulieu, L. (20/01/95) Contribution à la modélisation mathématique et numérique des écoulements diphasiques constitués d'un nuage de particules dans un écoulements de gaz., Habilitation Thesis, University of Paris VI, Paris, France.

[20] Sainsaulieu, L., Raviart, P. A. (1995) A nonconservative hyperbolic system modeling spray dynamics. Part 1: Solution of the Riemann problem., Mathematical Methods and Models in Applied Sciences 5, No.3,pp297-333.

[21] Smoller, J. (1983) Shock waves and reaction-diffusion equations, Springer-Verlag. 